\title{
Massive stars in globular clusters: drivers of chemical and dynamical evolution
}

\author{
Thibault Decressin $\dagger$ \\ Argelander Institute for Astronomy (AIfA), Auf dem Hügel 71, D-53121 Bonn, Germany \\ email: Thibaut.Decressin@unige.ch
}

\begin{abstract}
Massive stars have a strong impact on globular cluster evolution. First providing they rotate initially fast enough they can reach the break-up velocity during the main sequence and a mechanical mass-loss will eject matter from the equator at low velocity. Rotation-induced mixing will also bring matter from the convective core to the surface. From this ejected matter loaded in H-burning material a second generation of stars will born. The chemical pattern of these second generation stars are similar to the one observed for stars in globular cluster with abundance anomalies in light elements. Then during the explosion as supernovae the massive stars will also clear the cluster of the remaining gas. If this gas expulsion process acts on short timescale it can strongly modify the dynamical properties of clusters by ejecting preferentially first generation stars.
\end{abstract}

Keywords. stars: abundances, stars: evolution, stars: mass loss, globular clusters: general, stellar dynamics, methods: n-body simulations

\section{Introduction}

Globular clusters are self-gravitating aggregates of tens of thousands to millions of stars that have survived over a Hubble time. Many observations show that these objects are composed of multiple stellar populations. The first evidence rests on the chemical analysis that reveals large star-to-star abundance variations in light elements in all individual clusters studied so far, while the iron abundance stays constant (for a review see Gratton et al. 2004). These variations include the well-documented anticorrelations between $\mathrm{C}-\mathrm{N}$, O-Na, Mg-Al, Li-Na and F-Na (Kraft 1994; Carretta et al. 2007; Gratton et al. 2007; Pasquini et al. 2007; Carretta et al. 2010; Lind et al. 2009). H-burning at high temperature around $75 \times 10^{6} \mathrm{~K}$ is required to explain this global chemical pattern (Arnould et al. 1999; Prantzos et al. 2007). As the observed chemical pattern is present in low-mass stars both on the red giant branch (RGB) and at the turn-off that do not reach such high internal temperatures, the abundance anomalies must have been inherited at the time of formation of these stars.

Besides, deep photometric studies provide another indications for multiple populations in individual GCs with the discoveries of multiple giant and sub-giant branches or main sequences. In $\omega$ Cen a blue main sequence has been discovered (Bedin et al. 2004) that is presumably related to a high content in He (Piotto et al. 2005; Villanova et al. 2007). A triple main sequence has been discovered in NGC 2808 (Piotto et al. 2007). The additional blue sequences are explainable by a higher He content of the corresponding stars which shifts the effective temperatures towards hotter values. He-rich stars have also been proposed to explain the morphology of extended horizontal branch (hereafter HB) seen in many globular clusters (see e.g., Caloi \& D'Antona 2005). Whereas no direct

$\dagger$ Present address: Geneva Observatory, University of Geneva, 51 ch. des Maillettes, 1290 Versoix, Switzerland 


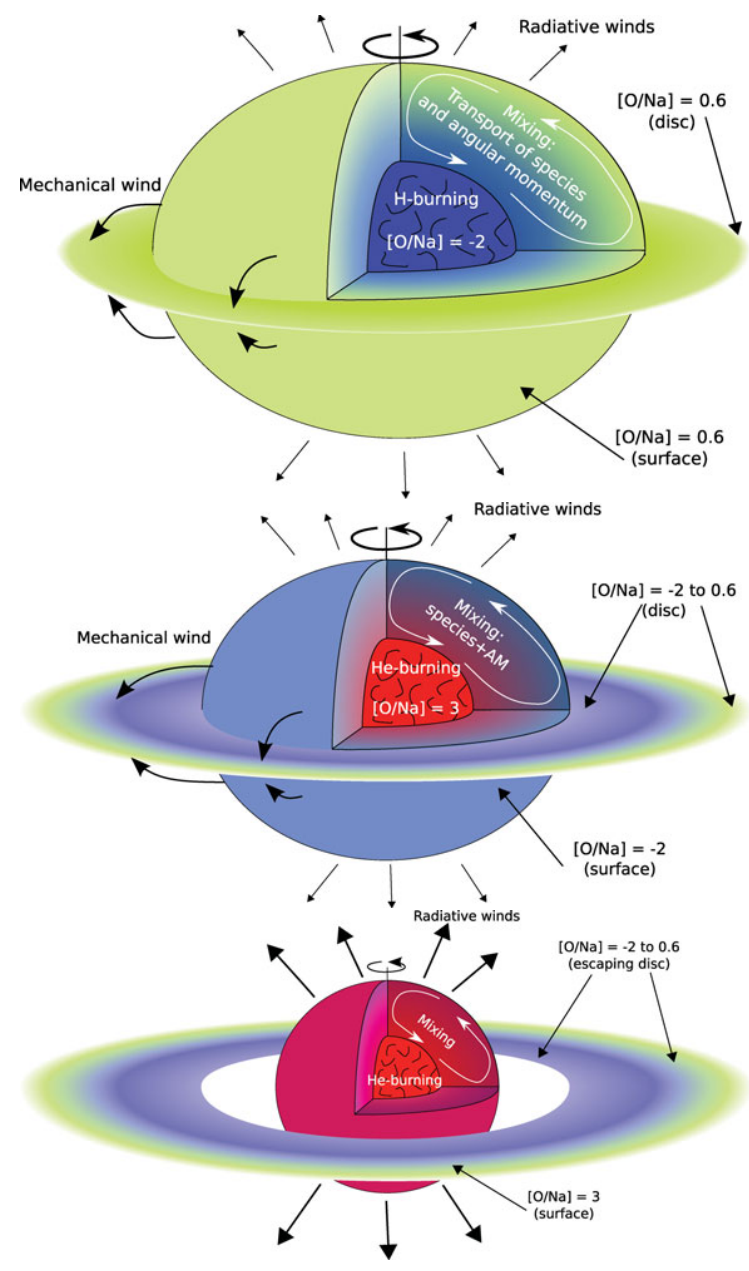

Figure 1. Schematic view of the evolution of fast rotating massive stars. The colours reflect the chemical composition of the various stellar regions and of the disc. (top) During the main sequence, a slow outflowing equatorial disc forms and dominates matter ejection with respect to radiative winds. (middle) At the beginning of central He-burning, the composition of the disc material spans the range in $[\mathrm{O} / \mathrm{Na}]$ observed today in low-mass cluster stars. The star has already lost an important fraction of its initial mass. (bottom) Due to heavy mass loss, the star moves away from critical velocity and does not supply its disc anymore; radiatively-driven fast wind takes over before the products of He-burning reach the stellar surface.

observational link between abundance anomalies and He-rich sequences has been found, this link is easily understood theoretically as abundance anomalies are the main result of $\mathrm{H}$ burning to He.

These observed properties lead to the conclusion that globular clusters born from giant gas clouds first form a generation of stars with the same abundance pattern as field stars. Then a polluting source enriches the intracluster-medium with H-burning material out of which a chemically-different second stellar generation forms. This scheme can explain at the same time the abundance anomalies in light elements and He-enrichment.

Two main candidates that reach the right temperature for H-burning have been proposed to be at the origin of the abundance anomalies (Prantzos \& Charbonnel 2006): (a) intermediate mass stars evolving on the thermal pulses along the asymptotic giant 
branch (hereafter TP-AGB), and (b) main sequence massive stars. After being first proposed by Cottrell \& Da Costa (1981) the AGB scenario has been extensively studied (see e.g., Ventura \& D'Antona 2008a,b, 2009, see Ventura, this volume) and has been seriously challenged by rotating AGB models that predict unobserved CNO enrichment in low-metallicity globular clusters (Decressin et al. 2009).

On the other hand, as being suggested by Brown \& Wallerstein (1993) and Wallerstein et al. (1987), massive stars can also pollute the interstellar medium (ISM) of a forming cluster (see Smith 2006; Prantzos \& Charbonnel 2006). In particular Decressin et al. (2007b) show that fast rotating massive stars (with a mass higher than $\sim 25 \mathrm{M}_{\odot}$ ) are good candidates for the self-enrichment of globular clusters (see $\S 2$ ). An alternative suggestion has been proposed by de Mink et al. (2009) to consider non-conservative mass-transfer from binaries stars. In the following we will only consider the consequences of the pollution by rotating massive stars.

\section{Chemical population of globular clusters}

In the wind of fast rotating massive stars (WFRMS) scenario sketched in Fig. 1, rotationally-induced mixing transports H-burning products (and hence matter with correct abundance signatures) from the convective core to the stellar surface. Providing initial rotation is high enough, the stars reach the break-up on the main sequence evolution. As a result a mechanical wind is launched from the equator that generates a disk around the star similar to that of Be stars (e.g. Townsend et al. 2004). Later, when He-burning products are brought to the surface, the star has already lost a high fraction of its initial mass and angular momentum, so that it no longer rotates at the break-up velocity. Matter is then ejected through a classical fast isotropic radiative wind. From the matter ejected in the disk, a second generation of stars may be created with chemical pattern in agreement with observations.

The slow winds ejected by massive stars have no Li has this fragile elements burn at $3.5 \times 10^{6} \mathrm{~K}$. The dilution of the slow winds (Li-free) with the pristine ISM (Li-rich) can explain the presence of $\mathrm{Li}$ in stars with abundance anomalies as observed in several clusters (see e.g., Pasquini et al. 2005; Lind et al. 2009; D’Orazi \& Marino 2010; D'Orazi et al. 2010). By taking this dilution into account we can reproduce the trends of the Li-Na anticorrelation observed in NGC 6752 as observed by (Pasquini et al. 2005) as well as the anticorrelation between $\mathrm{O}$ and $\mathrm{Na}$ (see Decressin et al. 2007a).

\section{Dynamical consequences for globular clusters}

Based on the determination of the composition of giant stars in 19 globular clusters by Carretta et al. (2009) the stars with abundance anomalies populate between 50 to $80 \%$ of the cluster stars.

How to produce such a high fraction of chemically peculiar stars? The main problem is that assuming a Salpeter (1955) IMF for the polluters, the accumulated mass of the slow winds ejected by the fast rotating massive stars would only provide $10 \%$ of the total number of low-mass stars. To match the observations thus requires either (a) a flat IMF with a slope of 0.55 instead of 1.35 (Salpeter's value), or (b) that $95 \%$ of the first generation stars have escaped the cluster (Decressin et al. 2007a). Here we first verify whether such a high loss of stars is possible, and which are the main processes that could drive it. 


\subsection{Dynamical evolution of globular clusters}

First we assume that the globular clusters display primordial mass segregation so that the massive stars are located at their center. Since we expect that the formation of the second generation of low-mass stars happens locally around individual massive stars (see Decressin et al. 2007a for more details), the second generation of stars will also be initially more centrally concentrated than the first generation. In such a situation, two competitive processes act in the clusters: the loss of stars in the outer cluster parts will first reduce the number of bound first generation stars; and the dynamical spread of the initially more concentrated second generation stars will stop this differential loss when the two populations are dynamically mixed.

Our analysis, based on the N-body models computed by Baumgardt \& Makino (2003) with the collisional Aarseth N-body code NBODY4 (Aarseth 1999), is presented in detail in Decressin et al. (2008).

As these models have been computed for a single stellar population, we apply the following process to mimic the formation of a cluster with two dynamically distinct populations: we sort all the low-mass stars $\left(M \leqslant 0.9 \mathrm{M}_{\odot}\right)$ according to their specific energy (i.e., their energy per unit mass). We define the second stellar generation as the stars with lowest specific energies, (i.e., those which are most tightly bound to the cluster due to their small central distance and low velocity). The number of second generation stars is given by having their total number representing $10 \%$ of the total number of low-mass stars. Initially, first generation stars show an extended distribution up to $40 \mathrm{pc}$ whereas the second generation stars (with low specific energy) are concentrated within $6 \mathrm{pc}$ around the centre.

Initially, as only stars of the first generation populate the outer part of the cluster owing to their high specific energy, only these first generation stars are lost in the early times. This lasts until the second generation stars migrate towards the outer part of the cluster. Depending on the cluster mass, it takes between 1 to 4 Gyr (about 2-3 relaxation times) to start losing second generation stars. Due to the time-delay to lose second generation stars, their fraction of second generation stars relative to first generation ones increases first. Then it tends to stay nearly constant as soon as the two distributions are similar. This fraction only increases by a factor of 2.5 over the cluster history. Therefore, these second generations stars can account for $25 \%$ of the low-mass stars present in the clusters. Compared to the observed ratios (50 to 80\% in 19 globular clusters) the internal dynamical evolution and the dissolution due to the tidal forces of the host Galaxy are not efficient enough. An additional mechanism is thus needed to expel the first generation stars more effectively.

\subsection{Gas expulsion}

As it operates early in the cluster history (a few million years after cluster formation at the latest), initial gas expulsion by supernovae is an ideal candidate for such a process. As the gas still present after the star formation is quickly removed, it ensues a strong lowering of the potential well of the cluster so that the outer parts of the cluster can become unbound.

Baumgardt \& Kroupa (2007) computed a grid of N-body models to study this process and its influence on cluster evolution by varying the free parameters: star formation efficiency, SFE, ratio between the half-mass and tidal radius, $r_{h} / r_{t}$, and the ratio between the timescale for gas expulsion to the crossing time, $\tau_{M} / t_{\text {Cross }}$. They show in particular that, in some extreme cases, the complete disruption of the cluster can be induced by gas expulsion. This process has also been used successfully by Marks et al. (2008) to explain 


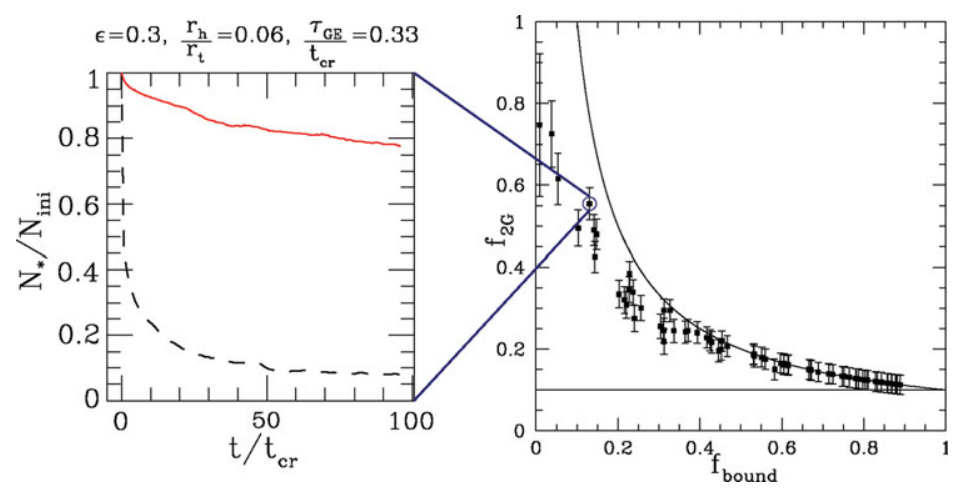

Figure 2. Left: Number of first (dotted line) and second (full line) generation stars relative to their initial number as a function of time for a cluster with the following initial properties: $\epsilon=0.3, r_{\mathrm{h}} / r_{\mathrm{t}}=0.06$ and $\tau_{G E} / t_{\mathrm{cr}}=0.33$. Right: Fraction of second-generation stars as a function of the final fraction of bound stars at the end of the computations of Baumgardt \& Kroupa (2007), i.e., after about 100 initial crossing times. Dashed lines indicate limiting cases where no second-generation stars are lost (upper) and no preferential loss of first-generation stars occurs (lower). Estimates of the statistical errors are also included based on the number of first, $N_{1}$, and second, $N_{2}$, generation stars bound to the cluster.

the challenging correlation between the central concentration and the mass function of globular clusters as found by De Marchi et al. (2007).

We have applied the same method as the one we used in $\S 2.2$ to the models of Baumgardt \& Kroupa (2007). Fig. 2 (left panel) shows that in the case of a cluster which loses around $90 \%$ of its stars, the ejection of stars from the cluster mostly concerns first generation ones. At the end of the computation only $7 \%$ of first generation stars remain bound to the cluster along with most of second generation stars. Therefore the number ratio between the second to first generation stars increases by a factor of 10: half of the population of low-mass stars still populating the cluster are second generation stars. The final fraction of second generation stars is strongly dependent of the initial properties of the cluster as shown in Fig. 2 (right panel) where this number is shown as a function of the fraction of star still bound to the cluster for the whole set of computation done by Baumgardt \& Kroupa (2007) (see Decressin et al. 2010 for more details).

Thus if globular clusters are born mass segregated, dynamical processes (gas expulsion, tidal stripping and two-body relaxation) can explain the number fraction of second generation stars with abundance anomalies. Similar conclusions have been reached by D'Ercole et al. (2008).

\section{Acknowledgements}

TD acknowledges support from the Swiss National Science Foundation (FNS) and from the "Programme National de Physique Stellaire" of CNRS/INSU, France.

\section{References}

Aarseth, S. J. 1999, PASP, 111, 1333

Arnould, M., Goriely, S. \& Jorissen, A. 1999, A\&A, 347, 572

Baumgardt, H. \& Kroupa, P. 2007, MNRAS, 380, 1589

Baumgardt, H. \& Makino, J. 2003, MNRAS, 340, 227

Bedin, L. R., Piotto, G., Anderson, J., Cassisi, S. et al. 2004, ApJ (Letters), 605, L125

Brown, J. A. \& Wallerstein, G. 1993, AJ, 106, 133

Caloi, V. \& D'Antona, F. 2005, A\&A, 435, 987 
Carretta, E., Bragaglia, A., Gratton, R. G., Recio-Blanco, A. et al. 2010, A $8 A$, 516, A55+

Carretta, E., Bragaglia, A., Gratton, R. G., Lucatello, S. et al. 2009, AछA, 505, 117

Carretta, E., Bragaglia, A., Gratton, R. G., Lucatello, S. et al. 2007, AछA, 464, 927

Cottrell, P. L. \& Da Costa, G. S. 1981, ApJ (Letters), 245, L79

De Marchi, G., Paresce, F., \& Pulone, L. 2007, ApJ (Letters), 656, L65

de Mink, S. E., Pols, O. R., Langer, N., \& Izzard, R. G. 2009, AÉA, 507, L1

Decressin, T., Baumgardt, H., Charbonnel, C., \& Kroupa, P. 2010, A\&A, 516, A73+

Decressin, T., Baumgardt, H., \& Kroupa, P. 2008, A\&A, 492, 101

Decressin, T., Charbonnel, C., \& Meynet, G. 2007a, A\&A, 475, 859

Decressin, T., Charbonnel, C., Siess, L., Palacios, A. et al. 2009, A\&BA, 505, 727

Decressin, T., Meynet, G., Charbonnel, C., Prantzos, N. et al. 2007b, A\&SA, 464, 1029

D'Ercole, A., Vesperini, E., D'Antona, F., McMillan, S. L. W., \& Recchi, S. 2008, MNRAS 391, 825

D'Orazi, V., Lucatello, S., Gratton, R., Bragaglia, A. et al. 2010, ApJ (Letters), 713, L1

D'Orazi, V. \& Marino, A. F. 2010, ApJ (Letters), 716, L166

Gratton, R., Sneden, C. \& Carretta, E. 2004, ARAA, 42, 385

Gratton, R. G., Lucatello, S., Bragaglia, A., Carretta, E. et al. 2007, A\&AA, 464, 953

Kraft, R. P. 1994, PASP, 106, 553

Lind, K., Primas, F., Charbonnel, C., Grundahl, F. et al. 2009, A\&A, 503, 545

Marks, M., Kroupa, P. \& Baumgardt, H. 2008, MNRAS, 386, 2047

Pasquini, L., Bonifacio, P., Molaro, P., Francois, P. et al. 2005, A\&\&A, 441, 549

Pasquini, L., Bonifacio, P., Randich, S., Galli, D. et al. 2007, A\&A, 464, 601

Piotto, G., Bedin, L. R., Anderson, J., King, I. R. et al. 2007, ApJ (Letters), 661, L53

Piotto, G., Villanova, S., Bedin, L. R., Gratton, R. et al. 2005, ApJ, 621, 777

Prantzos, N. \& Charbonnel, C. 2006, A\&A, 458, 135

Prantzos, N., Charbonnel, C., \& Iliadis, C. 2007, A\&A, 470, 179

Salpeter, E. E. 1955, ApJ, 121, 161

Smith, G. H. 2006, PASP, 118, 1225

Townsend, R. H. D., Owocki, S. P., \& Howarth, I. D. 2004, MNRAS, 350, 189

Ventura, P. \& D'Antona, F. 2008a, A\&A, 479, 805

Ventura, P. \& D'Antona, F. 2008b, MNRAS, 385, 2034

Ventura, P. \& D'Antona, F. 2009, A\& A, 499, 835

Villanova, S., Piotto, G., King, I. R., Anderson, J. et al. 2007, ApJ, 663, 296

Wallerstein, G., Leep, E. M., \& Oke, J. B. 1987, AJ, 93, 1137

\section{Discussion}

D. BAADE: Why does the evolution ends with the second generation, i.e. why isn't there a third generation

T. DECRESSin: In my models I consider that second generation stars consist only of lowmass stars. If this assumption is relaxed and the formation of massive second generation stars are allowed, you will run out of matter to form the third generation: a $60 \mathrm{M}_{\odot}$ star (first generation) can only produce a $30 \mathrm{M}_{\odot}$ star (second generation)

D. Bomans: Since we observe proto-globular clusters which have cleaned out their gas and have ages of $\sim 5-10 \times 10^{6} \mathrm{yr}$, what is the timescale of the formation of your second generation?

T. Decressin: The timescale for the formation of second generation stars need to be short to avoid that supernovae cleared out the slow winds of massive stars during the primordial gas expulsion process. It should be similar to the lifetime of massive polluters, i.e., a few Myr. 